\title{
Rapamycin inhibits ox-LDL-induced inflammation in human endothelial cells in vitro by inhibiting the mTORC2/PKC/c-Fos pathway
}

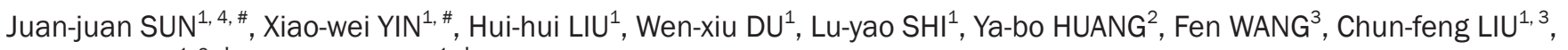 \\ Yong-jun $\mathrm{CAO}^{1,3, *}$, Yan-lin $\mathrm{ZHANG}^{1, *}$ \\ ${ }^{1}$ Department of Neurology, Second Affiliated Hospital of Soochow University, Suzhou 215004, China; ${ }^{2}$ Department of Neurosurgery, \\ First Affiliated Hospital of Soochow University, Suzhou 215006, China; ${ }^{3}$ Jiangsu Key Laboratory of Translational Research and Therapy \\ for Neuro-Psycho-Diseases and Institute of Neuroscience, Soochow University, Suzhou 215123, China; ${ }^{4}$ Department of Neurology, First \\ Hospital of Handan City, Handan 056002, China
}

\begin{abstract}
Rapamycin and its derivative possess anti-atherosclerosis activity, but its effects on adhesion molecule expression and macrophage adhesion to endothelial cells during atherosclerosis remain unclear. In this study we explored the effects of rapamycin on ox-LDLinduced adhesion molecule expression and macrophage adhesion to endothelial cells in vitro and the underlying mechanisms. Ox-LDL (6-48 $\mathrm{\mu g} / \mathrm{mL})$ dose-dependently increased the protein levels of two adhesion molecules, intercellular adhesion molecule-1 (ICAM1) and E-selectin, in human umbilical vein endothelial cells (HUVECs), whereas pretreatment with rapamycin (1-10 $\mu \mathrm{mol} / \mathrm{L})$ dosedependently inhibited ox-LDL-induced increase in the adhesion molecule expression and macrophage adhesion to endothelial cells. Knockdown of mTOR or rictor, rather than raptor, mimicked the effects of rapamycin. Ox-LDL $(100 \mu g / m L)$ time-dependently increased PKC phosphorylation in HUVECs, which was abolished by rapamycin or rictor siRNA. Pretreatment with PKC inhibitor staurosporine significantly reduced ox-LDL-stimulated adhesion molecule expression and macrophage adhesion to endothelial cells, whereas pretreatment with PKC activator PMA/TPA attenuated the inhibitory effect of rapamycin on adhesion molecule expression. Ox-LDL (100 $\mu \mathrm{g} / \mathrm{mL}$ ) time-dependently increased c-Fos levels in HUVECs, and pretreatment with rapamycin or rictor siRNA significantly decreased expression of c-Fos. Knockdown of c-Fos antagonized ox-LDL-induced adhesion molecule expression and macrophage adhesion to endothelial cells. Our results demonstrate that rapamycin reduces ox-LDL-stimulated adhesion molecule expression and macrophage adhesion to endothelial cells by inhibiting mTORC2, but not mTORC1, and mTORC2 acts through the PKC/c-Fos signaling pathway.
\end{abstract}

Keywords: atherosclerosis; rapamycin; endothelial cells; ox-LDL; ICAM-1; E-selectin; mTORC2; PKC; c-Fos; HUVECs

Acta Pharmacologica Sinica (2018) 39: 336-344; doi: 10.1038/aps.2017.102; published online 26 Oct 2017

\section{Introduction}

Atherosclerosis, a chronic inflammatory disease of the arterial wall $^{[1]}$, comprises a series of sequential and interrelated steps. It is well known that, in the initiation phase of atherosclerosis development, endothelial cells are stimulated by various risk factors, such as oxidized low-density lipoprotein (ox-LDL) ${ }^{[2]}$ and C-reactive protein $(\mathrm{CRP})^{[3]}$, to express adhesion and chemoattractant molecules that recruit inflammatory cells from the circulation to endothelial cells (ECs) and that encourage transmigration into the subendothelial space ${ }^{[4,5]}$. As adhesion

\footnotetext{
\#These authors contributed equally to this work.

${ }^{*}$ To whom correspondence should be addressed.

E-mail yongjuncao@126.com (Yong-jun CAO) zhangyanlin0012006@163.com (Yan-lin ZHANG)

Received 2017-03-29 Accepted 2017-07-09
}

is one of the critical steps in the initiation and progression of atherosclerosis ${ }^{[6]}$, cell adhesion intervention should be an effective strategy to alleviate atherosclerosis formation.

Rapamycin is in clinical trials as a treatment for cancer ${ }^{[7,8]}$ and has established uses in preventing vascular resteno$\operatorname{sis}^{[9-11]}$ and the immune rejection of transplanted organs ${ }^{[12]}$. Rapamycin inhibits mammalian target of rapamycin (mTOR), a conserved serine/threonine (Ser/Thr) protein kinase, known as mTOR complex 1 (mTORC1) and mTOR complex 2 (mTORC2) $^{[13]}$. The protein complex mTORC1 is composed of mTOR, mLST8, a regulatory-associated protein of mTOR (raptor), and a proline-rich Akt substrate of $40 \mathrm{kDa}$ (PRAS40) and is inhibited acutely (in minutes) by rapamycin ${ }^{[14-19]}$. The protein complex mTORC2 comprises mTOR, MLST8, mSin1, rapamycin-insensitive companion of $\mathrm{mTOR}$ (rictor), and pro- 
tein observed with rictor (protor) and is only altered after prolonged $(24 \mathrm{~h})$ treatment with rapamycin ${ }^{[14,20-27]}$. The mTORC2 protein complex regulates the phosphorylation or activity of Akt, glucocorticoid-inducible kinase 1 (SGK1), protein kinase $\mathrm{C}(\mathrm{PKC}) \mathrm{a}$, focal adhesion proteins, and small GTPases, and also controls cell survival and the actin cytoskeleton ${ }^{[20-27,28-32]}$.

Multiple groups have shown that rapamycin and its derivative possess anti-atherosclerosis functions via the reduction of vascular inflammation and the modulation of plaque composition $^{[33]}$, which affect the recruitment of monocytes into early lesions ${ }^{[34]}$, attenuate inflammation, and enhance the stability of atherosclerotic plaques ${ }^{[35]}$. A few studies have shown that rapamycin inhibits cell adhesion in colon cancer cells (HCT116) $^{[36]}$ and tumor cells ${ }^{[37]}$, indicating that rapamycin, an inhibitor of mTOR, may play important roles in the regulation of cell adhesion. However, whether rapamycin regulates cell adhesion in atherosclerosis and its associated underlying mechanism remain unknown.

\section{Materials and methods}

\section{Reagents and antibodies}

Ox-LDL was purchased from Yiyuan Biotechnologies (Guangzhou, China). Rapamycin was purchased from Sigma-Aldrich (St Louis, MO, USA), and phorbol 12-myristate 13-acetate (PMA)/12-O-tetradecanoylphorbol-13-acetate (TPA) and staurosporine were purchased from Biyuntian (Shanghai, China). The primary antibody against $\beta$-actin was obtained from Sigma, and the primary antibody against intercellular adhesion molecule-1 (ICAM-1) and F4/80 were purchased from Abcam (Cambridge, UK). Other primary antibodies were purchased from Santa Cruz Biotechnologies (Dallas, TX, USA). The cell culture reagents were obtained from Gibco (Grand Island, NY, USA).

\section{Cell culture and treatment}

Human umbilical vein endothelial cells (HUVECs) were purchased from Shanghai GeneChem Co Ltd (Shanghai, China). HUVECs and the murine macrophage cell line Raw264.7 were cultured in Dulbecco's modified Eagle's medium (DMEM) (Sigma-Aldrich) with $3.7 \mathrm{~g} / \mathrm{L} \mathrm{NaHCO}_{3}, 10 \%(v / v)$ fetal bovine serum $(\mathrm{FBS})$, and $1 \%(w / v)$ penicillin and streptomycin at $37^{\circ} \mathrm{C}$. HUVECs were preincubated with rapamycin at the indicated concentrations for $60 \mathrm{~min}$ before immediate exposure to ox-LDL $(100 \mu \mathrm{g} / \mathrm{mL})$.

\section{Cell viability measurement}

The Cell Counting Kit-8 (CCK-8) was used to measure cell viability according to the manufacturer's instructions. Briefly, HUVECs were seeded in a 96-well microplate at an appropriate density of cells/well and then pretreated with ox-LDL, rapamycin, mTOR siRNA, rictor siRNA, or raptor siRNA according to the indicated time course. Subsequently, CCK-8 solution $(10 \mu \mathrm{L} /$ well) was added to the wells, and the plate was incubated at $37^{\circ} \mathrm{C}$ for $1.5 \mathrm{~h}$; then, the absorbance was determined with a microplate reader (Tecan) at a wavelength of $490 \mathrm{~nm}$. The optical density value was reported as the per- centage of cell viability normalized to the control group .

\section{Western blot analysis}

Cells were washed with phosphate-buffered saline (PBS) and homogenized in ice-cold lysis buffer $(150 \mathrm{mmol} / \mathrm{L} \mathrm{NaCl}, 25$ $\mathrm{mmol} / \mathrm{L}$ Tris, $5 \mathrm{mmol} / \mathrm{L}$ ethylenediaminetetraacetic acid (EDTA), 1\% Nonidet P-40, pH 7.5) with protease inhibitor cocktail tablets (Roche Diagnostics, Penzberg, Germany). Protein concentrations were determined by bicinchoninic acid (BCA) protein assay kit (Pierce, Rockford, IL, USA). Protein samples were separated on $10 \%$ sodium dodecyl sulfatepolyacrylamide gels and transferred onto nitrocellulose membranes. Next, membranes were blocked in 5\% $(w / v)$ nonfat dry milk powder in $0.1 \%$ Tris-buffered saline/Tween 20 (TBST) for $1 \mathrm{~h}$ and then incubated with primary antibodies at optimized dilutions at $4{ }^{\circ} \mathrm{C}$ overnight. Membranes were then washed with TBST and incubated with horseradish peroxidase (HRP)-conjugated secondary antibodies for another $1 \mathrm{~h}$. Membranes were visualized using ECL chemiluminescence (Thermo Company, West Chester, PA, USA). The band densities were analyzed with ImageJ software (National Institutes of Health, Bethesda, MD, USA).

\section{Transient transfection}

The small interfering RNAs (siRNAs) targeting mTOR, rictor, raptor, and c-Fos were synthesized by GenePharma (Shanghai, China). The siRNAs were transfected using Lipofectamine 3000 (Invitrogen, Carlsbad, CA, USA). The knockdown efficiency was determined $24 \mathrm{~h}$ after transfection using Western blotting.

\section{Macrophage adhesion assay}

In order to evaluate the adherence of macrophages to the endothelium, Raw264.7 macrophages were preplated on $35-\mathrm{mm}$ dishes and cultured to $80 \%$ confluence. Simultaneously, HUVECs were pretreated with rapamycin/staurosporine for $1 \mathrm{~h}$ or $\mathrm{mTOR} / \mathrm{mTORC} 2 / \mathrm{c}-\mathrm{Fos}$ siRNA for $24 \mathrm{~h}$ before being subjected to ox-LDL for $24 \mathrm{~h}$. The culture medium, together with the macrophages, was transferred to the endothelial monolayer. After incubation at $37^{\circ} \mathrm{C}$ for $1 \mathrm{~h}$, nonadherent macrophages were removed by washing with PBS three times. Briefly, samples were permeabilized in PBST and blocked with $5 \%$ BSA, then incubated in primary antibody blocking solution overnight at $4{ }^{\circ} \mathrm{C}$. After washing, samples were incubated with Alexa Fluor ${ }^{\circledR}$ 555-conjugated secondary antibodies for $1 \mathrm{~h}$. DAPI was used to stain the cell nuclei. Subsequently, the number of macrophages adhering to the endothelial monolayers was counted under a Zeiss microscope (Axio Scope A1, Goettingen, Germany). The results are expressed as the mean number of cells per optical field at $20 \times$ magnification.

\section{Statistical analysis}

All data are presented as the mean \pm SEM. Statistical significance was analyzed using Student's $t$ test or one-way analysis of variance followed by a post-hoc analysis (Tukey's test) 
where applicable. The significance level was set at $P<0.05$.

\section{Results}

Rapamycin ameliorates ox-LDL-stimulated ICAM-1 and E-selectin expression and macrophage adhesion to HUVECs

The CCK-8 assay indicated that $25-100 \mu \mathrm{g} / \mathrm{mL}$ ox-LDL treatment for $24 \mathrm{~h}$ did not adversely affect cell viability (Figure 1A). To explore the optimal time for ox-LDL treatment in our study, we first evaluated the effects of different time points $(0,6,12,24$, and $48 \mathrm{~h})$ of ox-LDL treatment on the ICAM-1 and E-selectin expression levels in HUVECs by Western blot. As seen in Figure 1B-D, ox-LDL tended to time-dependently increase ICAM-1 and E-selectin levels; these proteins reached their peak levels at $24 \mathrm{~h}$ but decreased after $24 \mathrm{~h}$. After $24 \mathrm{~h}$ of treatment, ox-LDL enhanced ICAM-1 and E-selectin levels by approximately 1.7 - and 2.2-fold, respectively. Therefore, the treatment of HUVECs with ox-LDL for $24 \mathrm{~h}$ was chosen for the subsequent experiments.

The CCK-8 assay showed that cell viability was not influenced by 1.25-10 $\mu \mathrm{mol} / \mathrm{L}$ rapamycin treatment for $24 \mathrm{~h}$ (Figure $1 \mathrm{E})$. HUVECs were pretreated with rapamycin at different concentrations $(1.25,2.5,5$, or $10 \mu \mathrm{mol} / \mathrm{L})$ for $1 \mathrm{~h}$. Cells were then exposed to ox-LDL $(100 \mu \mathrm{g} / \mathrm{mL})$ for $24 \mathrm{~h}$ in the presence or absence of rapamycin. The key mediators involved in enhancing endothelial cell-macrophage interaction, ICAM-1 and E-selectin, were tested. As shown in the groups with rapamycin pretreatment (Figure $1 \mathrm{~F}-\mathrm{H}$ ), which did not influence ICAM-1 and E-selectin expression itself, rapamycin significantly antagonized ox-LDL-induced ICAM-1 and E-selectin increases in a dose-dependent manner.

We also investigated whether rapamycin affected the adhesion of macrophages to HUVECs. The number of adherent macrophages in groups that were subjected to ox-LDL pretreatment was remarkably higher than those exposed to vehicle treatment alone (control). Ox-LDL-induced macrophage adhesion to HUVECs was reduced in the presence of rapamycin (Figure 1I and J).

Rapamycin reduces ox-LDL-stimulated ICAM-1 and E-selectin expression and macrophage adhesion to HUVECs possibly via the inhibition of mTOR

In order to examine whether rapamycin inhibits adhesion
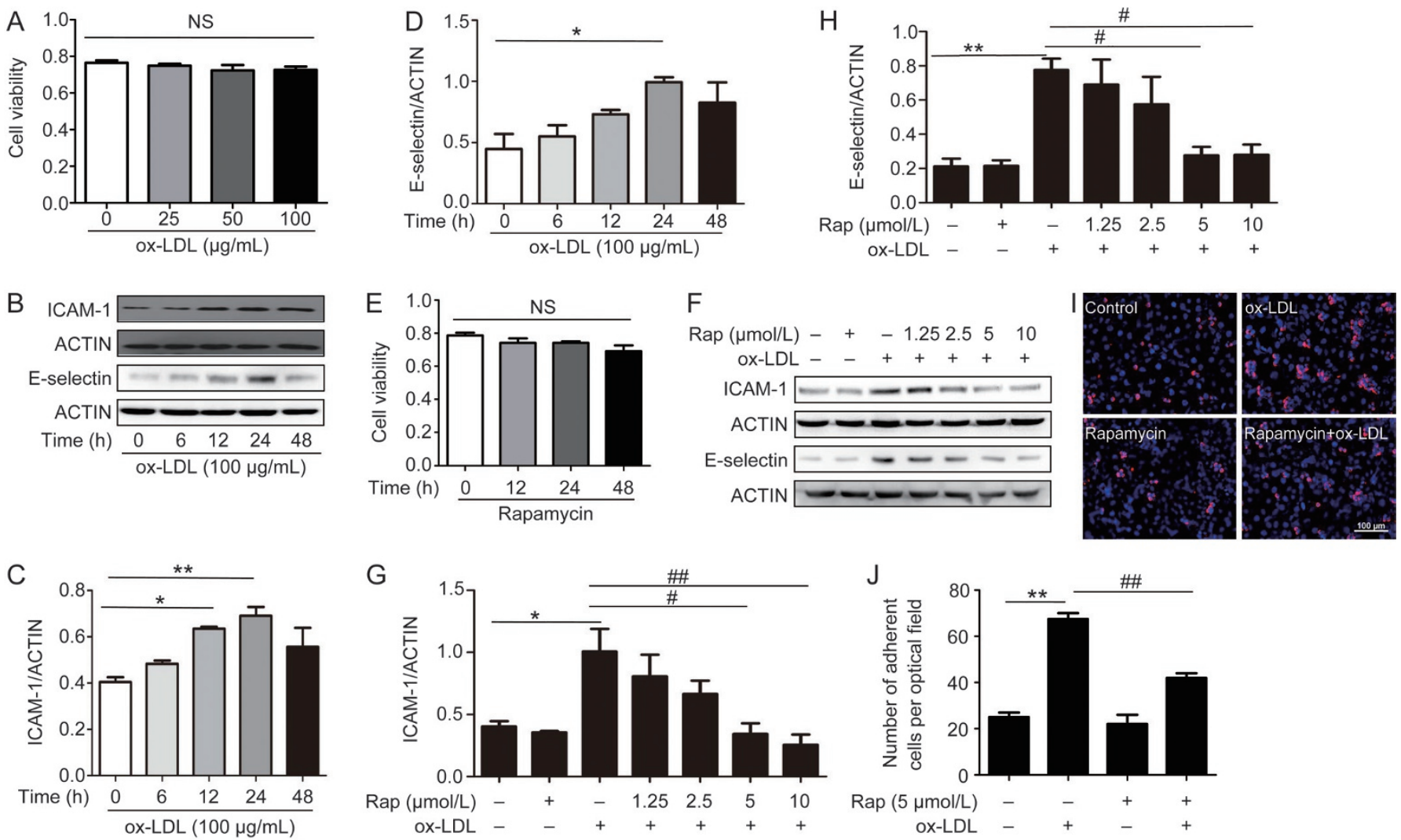

Figure 1. Rapamycin reduces ox-LDL-induced ICAM-1 and E-selectin expression and macrophage adhesion to endothelial cells. CCK-8 assay for cell viability after treatment with ox-LDL or rapamycin by time course (A, E). NS = not significant. (B-D) Changes in ICAM-1 and E-selectin protein expression in endothelial cells subjected to $100 \mu \mathrm{g} / \mathrm{mL}$ ox-LDL treatment for $0,6,12,24$, and $48 \mathrm{~h}$. (F-H) Cells were pretreated with $1.25,2.5,5$, and $10 \mu \mathrm{mol} / \mathrm{L}$ rapamycin for $1 \mathrm{~h}$, followed by $100 \mu \mathrm{g} / \mathrm{mL}$ ox-LDL treatment for $24 \mathrm{~h}$. ICAM-1 and E-selectin protein expression levels are indicated by WB. Actin was used as a loading control. (I-J) Representative optical fields (I) and quantitative analysis $(J)$ of the numbers of macrophages adhering to endothelial cells. Red, macrophage cells immunopositive for F4/80. Blue, nuclear counterstain. Representative images of the adhesion assay are shown at $20 \times$ magnification. ${ }^{*} P<0.05,{ }^{* *} P<0.01$ vs control. ${ }^{\#} P<0.05,{ }^{\# \#} P<0.01$ vs ox-LDL group. Mean \pm SEM. $n=3-4$. Scale bar=100 $\mu \mathrm{m}$. 
molecule expression and macrophage adhesion to HUVECs by inhibiting mTOR, mTOR siRNA was used to silence mTOR. The CCK-8 assay showed that mTOR siRNA treatment of HUVECs for $0,12,24$, and $48 \mathrm{~h}$ did not adversely affect cell viability (Figure 2A). Western blot analyses revealed that mTOR protein expression was decreased by approximately $66 \%$ in HUVECs with mTOR siRNA transfection (Figure $2 \mathrm{~B}$ and $\mathrm{C}$ ). Similar to rapamycin, siRNA-inhibited mTOR decreased levels of ICAM-1 and E-selectin after ox-LDL stimulation (Figure 2D-F) by approximately $51 \%$ and $65 \%$, respectively. Functionally, mTOR knockout decreased the ox-LDL-stimulated adhesion of macrophages to HUVECs by approximately $65 \%$ (Figure $2 \mathrm{G}$ and $\mathrm{H}$ ), whereas we observed a slight reduction in basal ICAM-1 and E-selectin expression, as well as in the number of macrophages adhering to HUVECs, in mTOR-deficient cells.

Rapamycin reduces ox-LDL-stimulated ICAM-1 and E-selectin expression and macrophage adhesion to HUVECs possibly via the inhibition of mTORC2 but not of mTORC1

Next, siRNA specific to raptor, an essential component of mTORC1, was utilized to determine whether mTORC1 was the target of rapamycin. The CCK-8 assay showed that raptor siRNA treatment for $0,12,24$, and 48 h did not adversely affect cell viability (Figure 3A). The knockdown efficiency of raptor siRNA reached approximately $85 \%$, as determined by raptor protein expression (Figure $3 \mathrm{~B}$ and C). Surprisingly, levels of ICAM-1 and E-selectin (Figure 3D-F) and the numbers of adherent macrophages (Figure 3G and $\mathrm{H}$ ) with or without oxLDL treatment after preincubation and with or without raptor siRNA treatment for $24 \mathrm{~h}$ were not significantly affected.

The CCK-8 assay showed that cell viability was not influenced by rictor siRNA treatment for 0, 12, 24, and $48 \mathrm{~h}$ (Figure 3I). We used the RNA interfering technique to specifically decrease rictor expression, as shown in Figure 3J and $\mathrm{K}$. Interestingly, downregulation of Rictor suppressed ox-LDLinduced ICAM-1 and E-selectin expression by approximately $82 \%$ and $47 \%$, respectively (Figure 3L-O). Functionally, knockout of rictor ameliorated ox-LDL-stimulated adhesion of macrophages to HUVECs by approximately 62\% (Figure 3P and $Q)$, whereas treatment with rictor siRNA itself did not significantly affect ICAM-1 and E-selectin levels or the number of macrophages adhering to HUVECs (Figure 3P, Q).

Inhibition of mTORC2 reduces ICAM-1 and E-selectin expression and macrophage adhesion to HUVECs by inhibiting the activation of PKC

Ox-LDL time-dependently induced an increase in PKC phos-
A
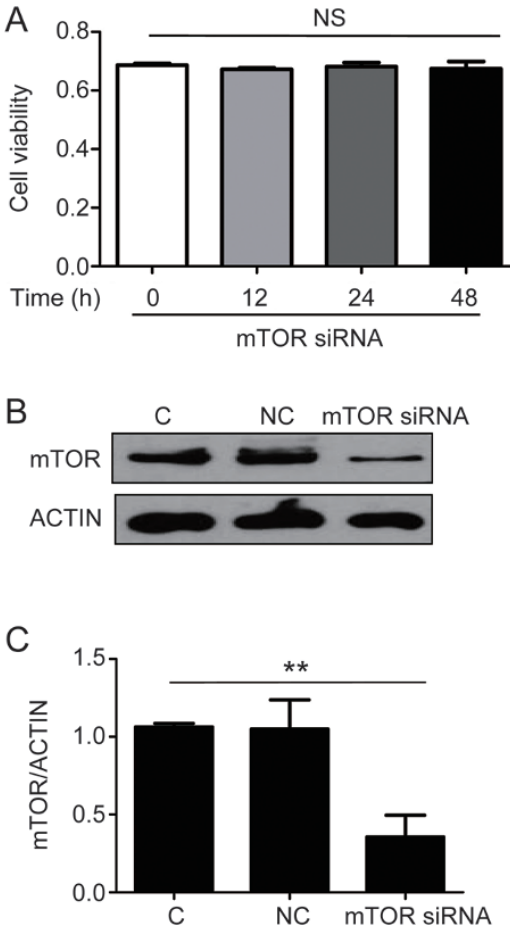

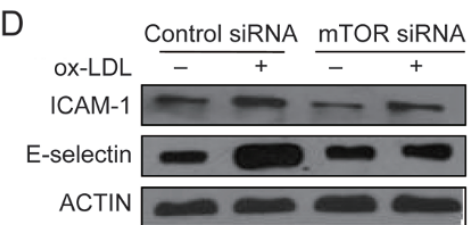

E
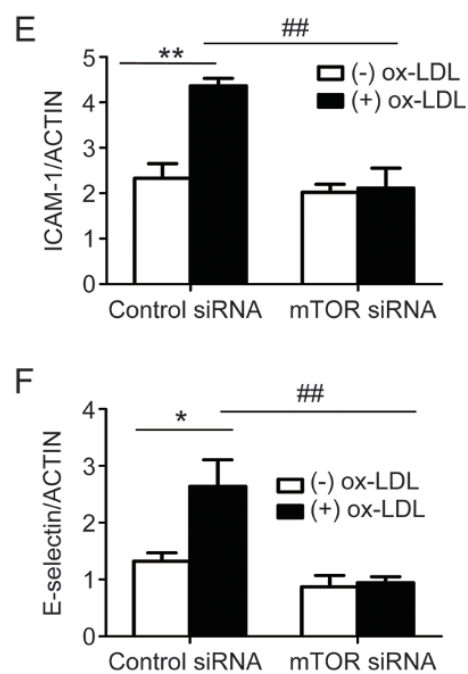
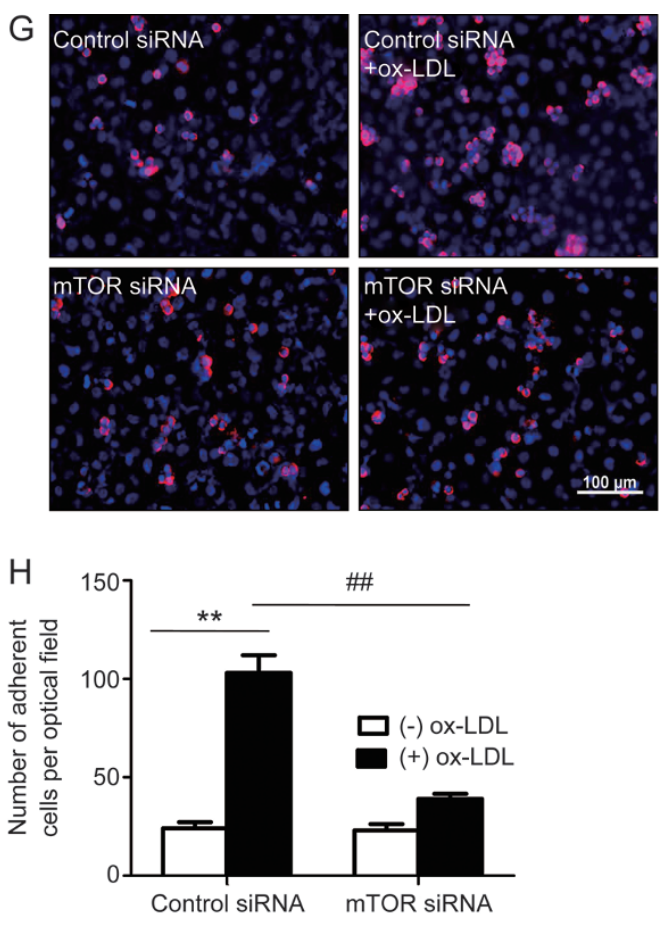

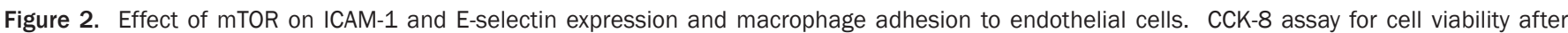

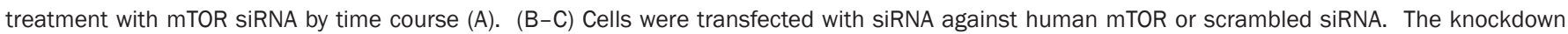

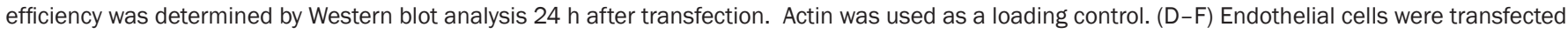

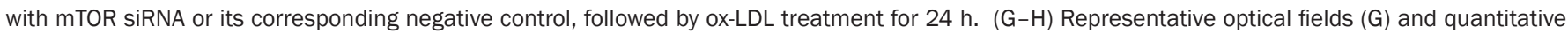

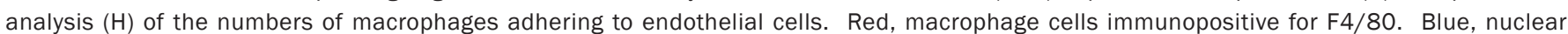

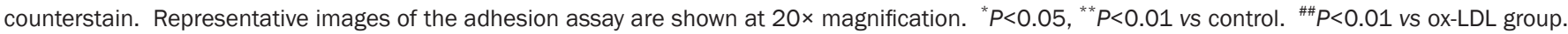
Mean \pm SEM. $n=3-4$. Scale bar $=100 \mu \mathrm{m}$. 
A
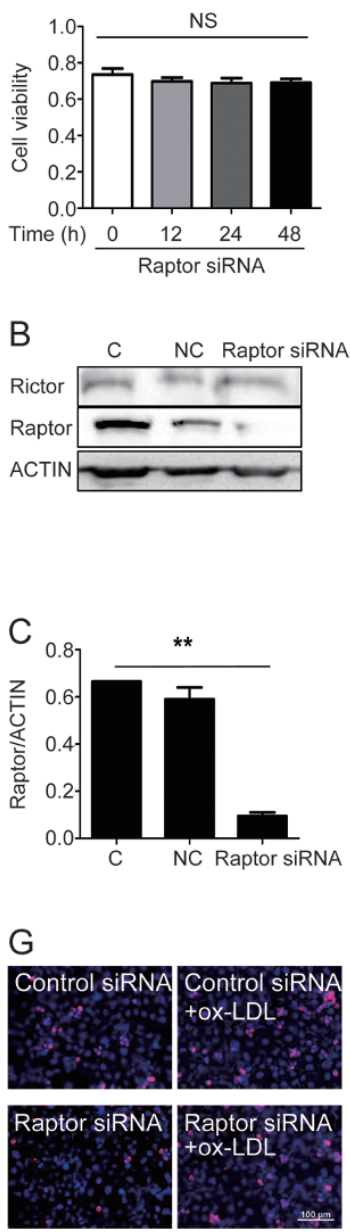

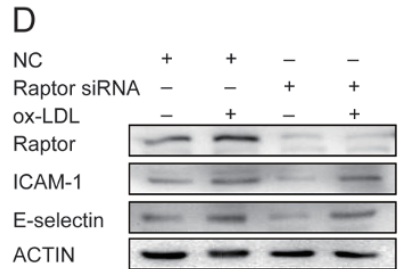

ACTIN
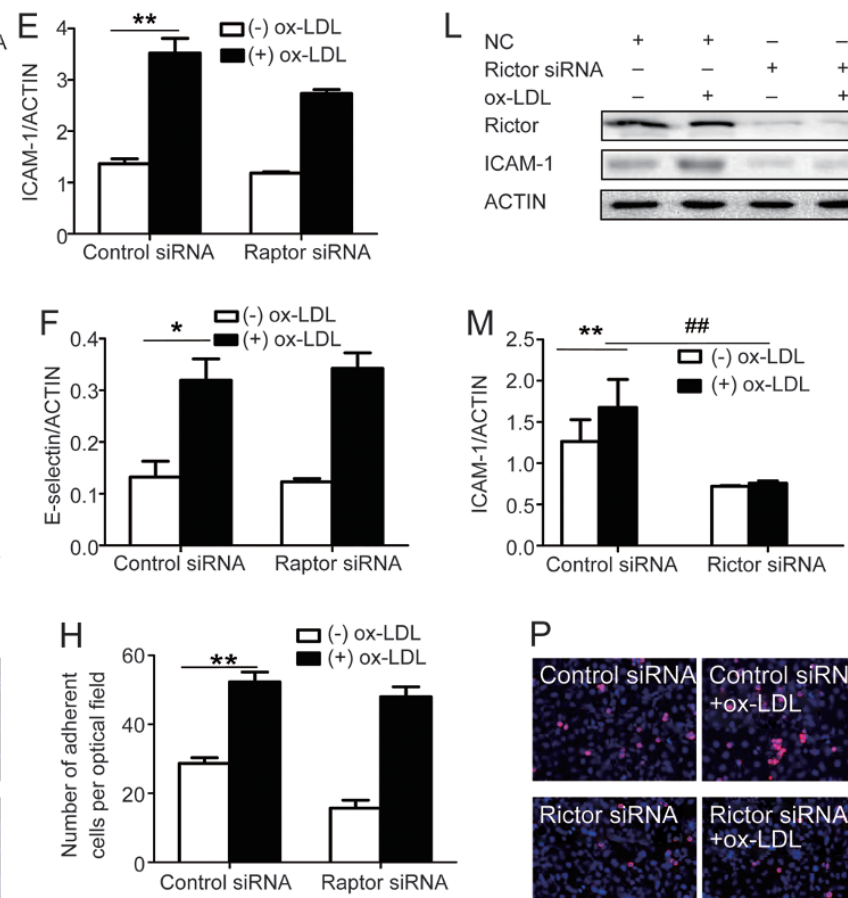

I

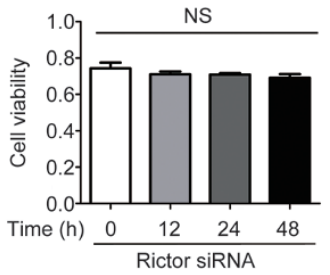

L
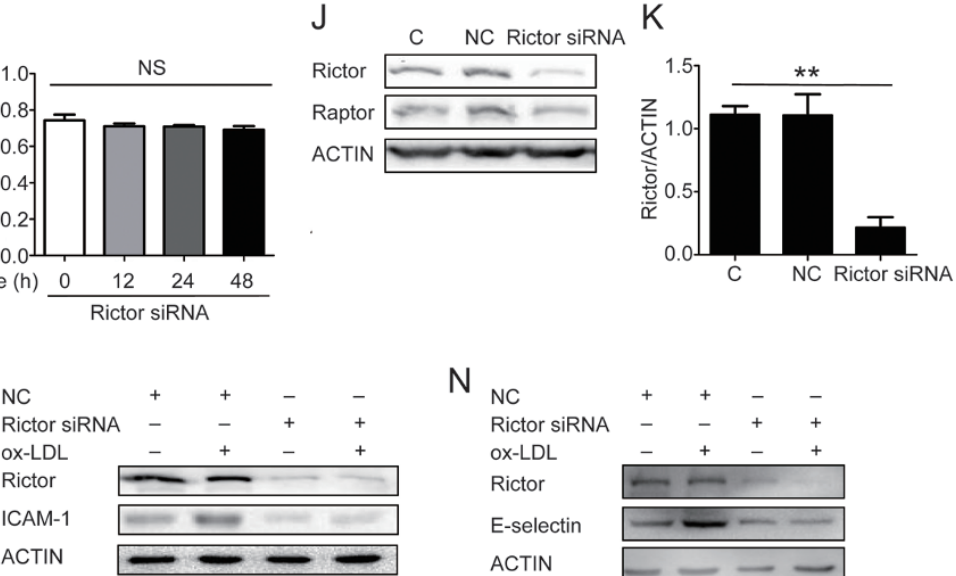

$\mathrm{N}_{\mathrm{NC}}$
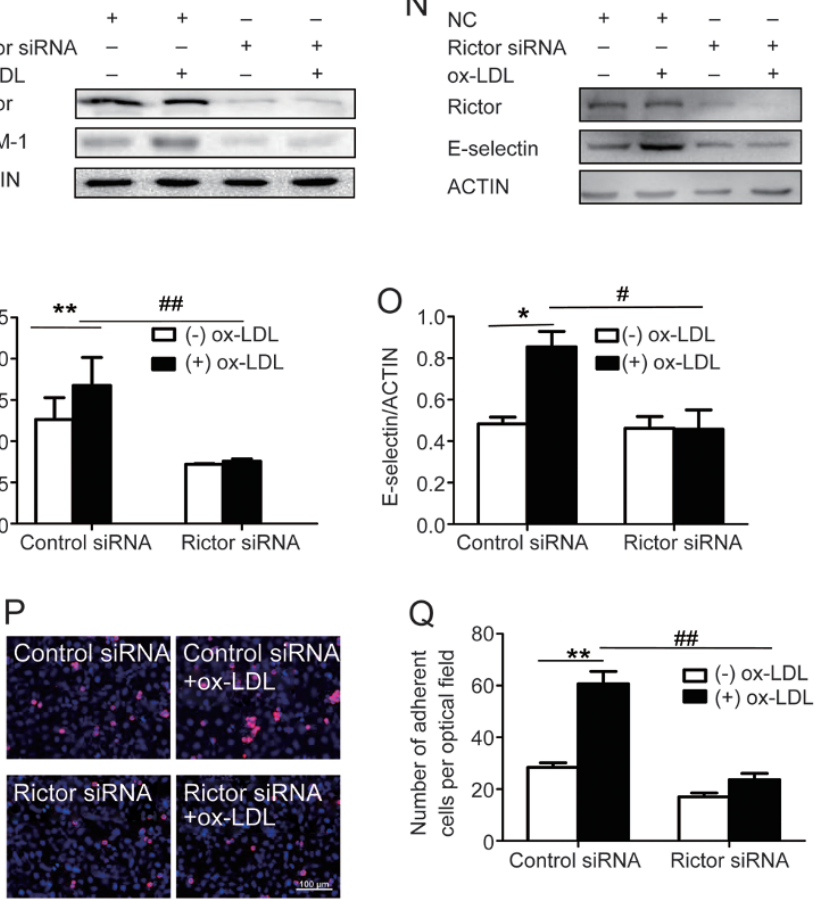

Figure 3. Effect of mTORC1 and mTORC2 on ICAM-1 and E-selectin expression and macrophage adhesion to endothelial cells. CCK-8 assay for cell viability after treatment with raptor siRNA or rictor siRNA by time course $(A, I)$. (B-C) Cells were transfected with siRNA against human mTORC1 or scrambled siRNA. The knockdown efficiency was determined by Western blot analysis $24 \mathrm{~h}$ after transfection. Actin was used as a loading control. (D-F) Endothelial cells were transfected with mTORC1 siRNA or its corresponding negative control, followed by ox-LDL treatment for $24 \mathrm{~h}$. (G-H) Representative optical fields $(G)$ and quantitative analysis $(H)$ of the numbers of macrophages adhering to endothelial cells. Red, macrophage cells immunopositive for F4/80. Blue, nuclear counterstain. Representative images of the adhesion assay are shown at $20 \times$ magnification. (J-K) Cells were transfected with siRNA against human mTORC2 or scrambled siRNA. The knockdown efficiency was determined by Western blot analysis $24 \mathrm{~h}$ after transfection. Actin was used as a loading control. (L-O) Endothelial cells were transfected with mTORC2 siRNA or its corresponding negative control, followed by ox-LDL treatment for $24 \mathrm{~h}$. (P-Q) Representative optical fields (P) and quantitative analysis (Q) of the numbers of macrophages adhering to endothelial cells. Red, macrophage cells immunopositive for F4/80. Blue, nuclear counterstain. Representative images of the adhesion assay are shown at $20 \times$ magnification. ${ }^{*} P<0.05,{ }^{* * *} P<0.01$ vs control. ${ }^{*} P<0.05,{ }^{\# \#} P<0.01$ vs ox-LDL group. Mean \pm SEM. $n=3-4$. Scale bar $=100 \mu \mathrm{m}$.

phorylation in endothelial cells (Figure 4A). The maximal effect was achieved 30 min after ox-LDL treatment, with a rapid decline in ox-LDL-induced PKC phosphorylation at 60 and $120 \mathrm{~min}$. The ratio of phosphorylated PKC to total PKC in ox-LDL $(100 \mu \mathrm{g} / \mathrm{mL}, 30 \mathrm{~min})$-treated cells was increased approximately 3.2-fold over the control cells (Figure 4A). This effect was abolished by rapamycin $(5 \mu \mathrm{mol} / \mathrm{L})$ pretreatment (Figure 4B). A similar effect was observed in mTORC2 siRNApretreated cells (Figure 4C). Treatment with rapamycin or mTORC2 siRNA itself did not alter this ratio.

In order to determine the role of the PKC pathway in the regulation of adhesion molecule expression, we inhibited PKC with the PKC inhibitor staurosporine. As shown in Figure
$4 \mathrm{D}-\mathrm{H}$, pretreatment with staurosporine reduced ox-LDL-stimulated ICAM-1 and E-selectin expression by approximately $58 \%$ and $67 \%$, respectively. Simultaneously, the number of macrophages adhering to HUVECs decreased by approximately $60 \%$ compared with the ox-LDL group. To further confirm the role of PKC, we used PMA/TPA, an activator of PKC. As shown in Figure 4I-K, treatment with PMA/TPA attenuated the inhibitory effect of rapamycin on ICAM-1 and E-selectin expression.

C-Fos may be involved in mTORC2-mediated ICAM-1 and E-selectin expression and macrophage adhesion to HUVECs We also observed that ox-LDL markedly induced c-Fos but 
A
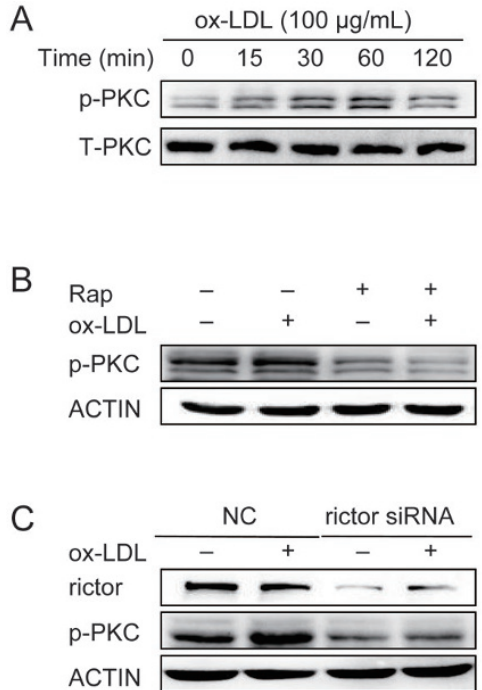

D
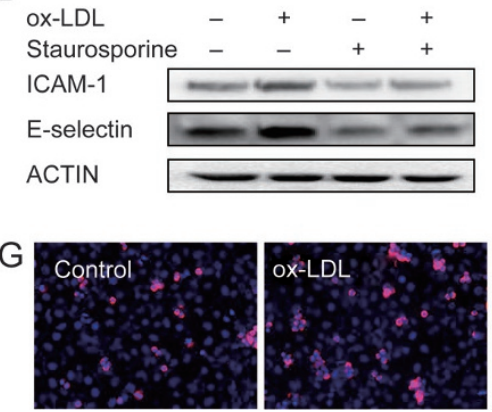

E

\section{E}

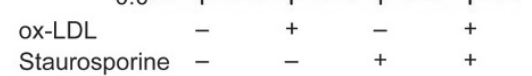

F

ox-LDL Staurosporin

\section{I}
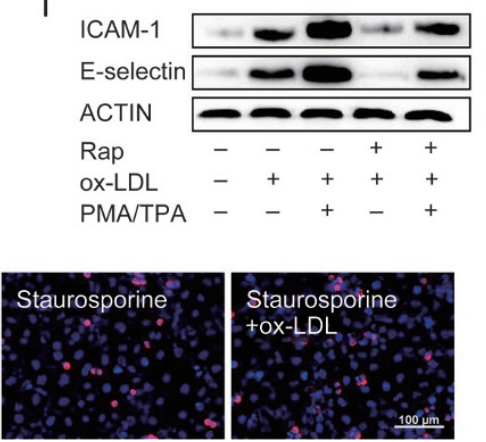

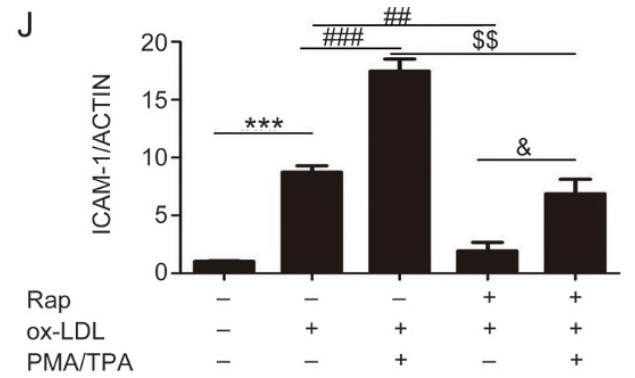

K
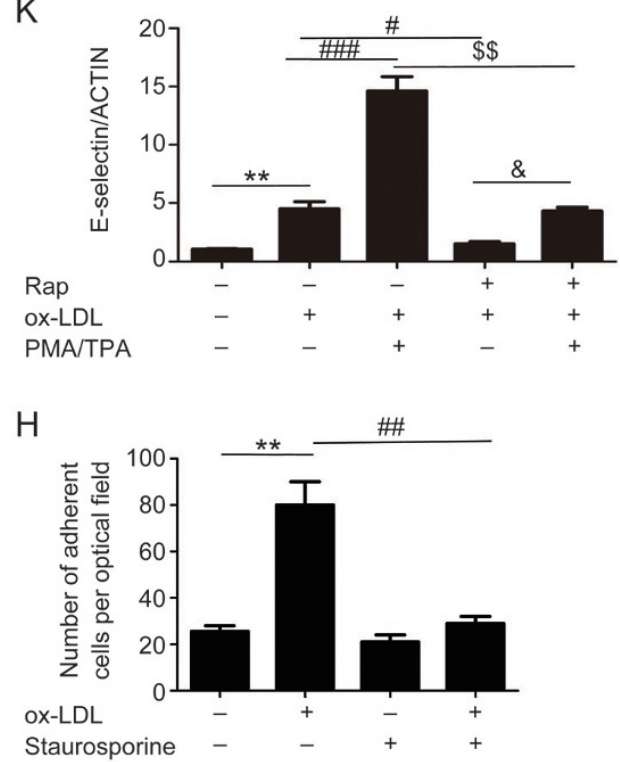

Figure 4. Effect of PKC on ICAM-1 and E-selectin expression and macrophage adhesion to endothelial cells. (A) Phosphorylation levels of PKC in endothelial cells stimulated with ox-LDL $(100 \mu \mathrm{g} / \mathrm{mL})$ for $0.25,0.5,1$, and $2 \mathrm{~h}$. (B-C) Effects of rapamycin and mTORC2 siRNA on the phosphorylation levels of PKC. Cells were pretreated with rapamycin for $1 \mathrm{~h}$ or mTOR siRNA for $24 \mathrm{~h}$, followed by ox-LDL treatment for $24 \mathrm{~h}$. (D-H) Effects of PKC inhibitors on ICAM-1 and E-selectin protein levels (D-F) and macrophage adhesion to endothelial cells (G-H). (I-K) Effects of PKC activators on ICAM-1 and E-selectin protein levels. The results were normalized to the control value. Data are expressed as the mean \pm SEM. $n=3-4$. ${ }^{*} P<0.05,{ }^{* *} P<0.01$ vs control. ${ }^{\#} P<0.05,{ }^{\#} P<0.01$ vs ox-LDL group. ${ }^{\$ \$} P<0.001$ vs PMA/TPA plus ox-LDL group. ${ }^{\&} P<0.05$ vs rapamycin plus ox-LDL group. Mean \pm SEM. $n=4-5$. Scale bar $=100 \mu \mathrm{m}$.

not phosphorylated (p)-c-Jun protein expression in endothelial cells (Figure 5A and B); this response occurred in a timedependent manner. The level of c-Fos protein expression in HUVECs was significantly increased at $24 \mathrm{~h}$ and $48 \mathrm{~h}$ after oxLDL treatment $(100 \mu \mathrm{g} / \mathrm{mL})$, and a smaller, but not significant, change in c-Fos protein expression was detected at 6 and 12 $\mathrm{h}$ after ox-LDL exposure. To investigate the role of c-Fos, we inhibited c-Fos using c-Fos siRNA. The Western blot results showed that c-Fos siRNA pretreatment significantly inhibited ox-LDL-induced ICAM-1 and E-selectin expression (Figure 5C-E). Moreover, the number of macrophages adhering to HUVECs decreased compared with exposure to ox-LDL alone (Figure 5F and G).

In order to further confirm the role of c-Fos in our study, we pretreated with rapamycin/rictor siRNA (Figure 5H-K) and the PKC inhibitor staurosporine (Figure 5L). We found that both rapamycin/rictor siRNA and staurosporine blocked oxLDL-stimulated c-Fos protein expression.

\section{Discussion}

Atherosclerosis, which is a major cause of cardiovascular disease, is a serious worldwide health concern. Adhesion is a critical step in the progression of atherosclerosis ${ }^{[6]}$. Accordingly, the disturbance of macrophage adhesion to HUVECs may interrupt atherosclerosis progression. Many studies have shown that rapamycin has anti-atherosclerosis functions ${ }^{[33,35]}$. However, whether rapamycin regulates cell adhesion in atherosclerosis and the underlying molecular mechanisms remain elusive. Here, we show that rapamycin attenuates ox-LDLtriggered ICAM-1 and E-selectin expression, as well as macrophage adhesion to HUVECs, by inhibiting mTORC2, but not mTORC1. Mechanistically, mTORC2 acts through the PKC/ c-Fos signaling pathway.

Increasing numbers of studies have shown that rapamycin, or its analogue, inhibits cell adhesion in cancer cells ${ }^{[36,37]}$ and endothelial cells ${ }^{[38]}$. However, one study has also shown that rapamycin does not affect adhesion molecule expres- 
A
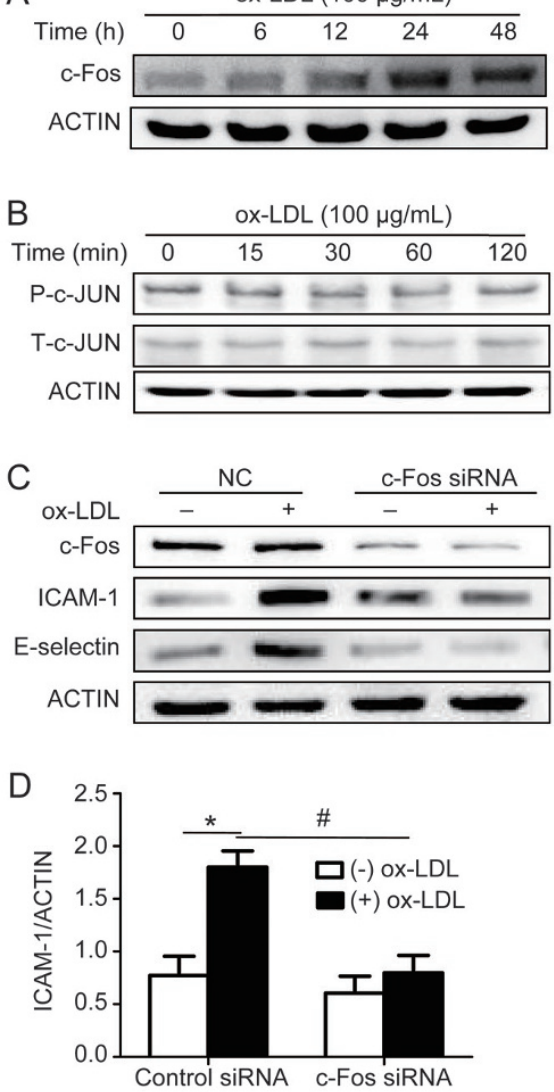
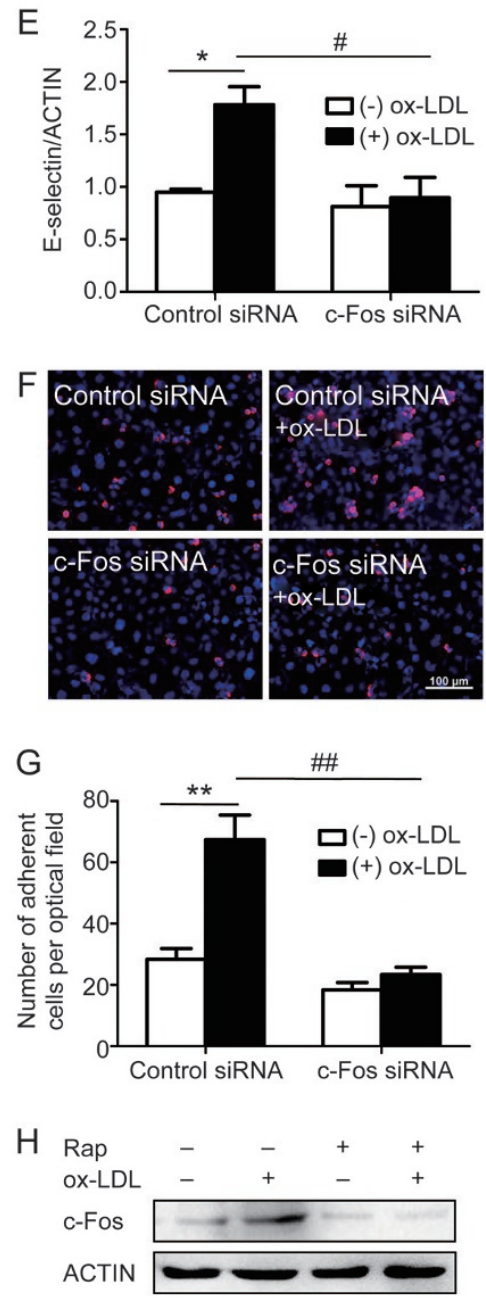
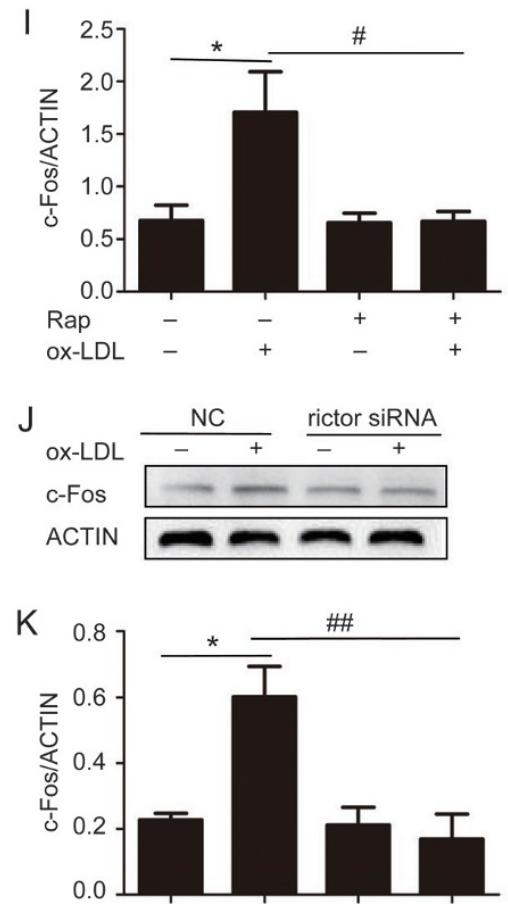

L

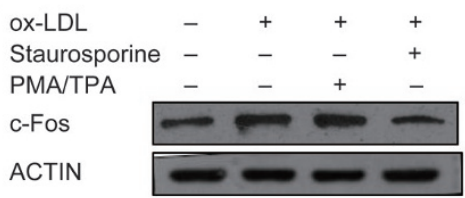

Figure 5. Effect of c-Fos on ICAM-1 and E-selectin expression and macrophage adhesion to endothelial cells. Levels of c-Fos (A) and p-c-Jun (B) in endothelial cells stimulated with ox-LDL $(100 \mu \mathrm{g} / \mathrm{mL})$ for $6,12,24$, and $48 \mathrm{~h}(\mathrm{~A})$ and $0.25,0.5,1$, and $2 \mathrm{~h}(\mathrm{~B})$. (C-G) Effects of c-Fos siRNA on ICAM1 and E-selectin protein levels (C-E) and macrophage adhesion to endothelial cells ( $F-G)$. ( $H-K)$ Effects of rapamycin (H-I) and mTORC2 siRNA ( $J-$ $\mathrm{K}$ ) on c-Fos levels. Cells were pretreated with rapamycin for $1 \mathrm{~h}$ or mTORC2/c-Fos siRNA for $24 \mathrm{~h}$, followed by ox-LDL treatment for $24 \mathrm{~h}$. (L) Effects of $\mathrm{PKC}$ inhibitors on c-Fos levels. The results were normalized to the control values. Data are expressed as the mean \pm SEM. $n=4$. ${ }^{*} P<0.05$ vs control. ${ }^{\#} P<0.05,{ }^{\# \#} P<0.01$ vs ox-LDL group. Scale bar $=100 \mu \mathrm{m}$.

sion in macrovascular and microvascular endothelial cells ${ }^{[39]}$, although this finding remains controversial. Here, we aimed to determine whether rapamycin inhibits cell adhesion in atherosclerosis and to investigate the underlying mechanism. In the present study, we observed that rapamycin suppresses oxLDL-stimulated ICAM-1 and E-selectin expression and macrophage adhesion to HUVECs (Figure 1F-J) in a concentrationdependent manner.

It is well known that mTORC1 is inhibited acutely (in minutes) by rapamycin, while mTORC2 is only affected after longer treatment with rapamycin ${ }^{[22]}$. Because we found that rapamycin inhibits ox-LDL-induced ICAM-1 and E-selectin expression and macrophage adhesion to HUVECs, we thus hypothesized that the rapamycin-sensitive complex, mTORC1, regulates this process. Surprisingly, we found that the disruption of mTORC2, but not the disruption of mTORC1, inhibits ox-LDL-stimulated ICAM-1 and E-selectin expression, as well as macrophage adhesion to HUVECs, implicating mTORC2 as the target of rapamycin (Figure 3). This is consistent with recent finding ${ }^{[40]}$ showing that rapamycin reduced vascular cell adhesion molecule 1 (VCAM-1) expression by inhibiting mTORC2. However, it has also been reported that both mTORC1 and mTORC2 are involved in the regulation of cell adhesion in a panel of tumor cell lines ${ }^{[37]}$. This is likely related to the different cell types or approaches used in the different studies. To confirm whether mTOR/mTORC2 is essential for this process, determining the effect of overexpression of $\mathrm{mTOR} / \mathrm{mTORC} 2$ on adhesion molecule expression and the number of macrophages adhering to HUVECs was needed in our study, but we were unable to perform these experiments due to laboratory restrictions. Further studies will be necessary to demonstrate the effect of the overexpression of mTOR/ mTORC2 on adhesion molecule expression and the number of macrophages adhering to HUVECs. 
Other studies have also shown that PKC regulates cell adhesion; enzymatically modified LDL (E-LDL) induced endothelial cell adhesion, promoted the transposition and activation of PKC, inhibited the expression of $\mathrm{I} \kappa \mathrm{Ba}$, and enhanced the expression of ICAM-1 ${ }^{[41]}$. We also noted that the inhibition of $\mathrm{mTOR}$ by rapamycin or the downregulation of rictor decreased the phosphorylation of PKC (Figure 4B and C). Early studies have suggested that the ablation of mTORC2 components (rictor, Sin1, or mTOR) abolishes phosphorylation of the turn motif (TM) of PKCa ${ }^{[42]}$ and that mTOR, Sin1, and rictor, components of $\mathrm{mTORC} 2$, are required for the phosphorylation of Akt and conventional PKC ${ }^{[43]}$. Cells treated with PMA/TPA, an activator of PKC, conferred resistance to rapamycin, and cell adhesion was rescued. Consistently, staurosporine, an inhibitor of PKC, potently inhibited ox-LDLstimulated cell adhesion. Our data suggest that PKC is essential for rapamycin inhibition of ox-LDL-induced ICAM-1 and E-selectin expression.

Previous studies ${ }^{[4]}$ have shown that c-Fos suppresses ovarian cancer progression by changing adhesion. Similarly, we observed that silencing c-Fos inhibited ox-LDL-induced adhesion molecule expression and macrophage adhesion to HUVECs; in addition, downregulation of mTORC2 or PKC reduced c-Fos expression in HUVECs. These findings suggest that mTORC2 acts through the PKC/c-Fos signaling pathway to affect ox-LDL-induced adhesion molecule expression and macrophage adhesion to HUVECs. Further research will be needed to clarify the downstream mechanism by which c-Fos contributes to adhesion molecule expression during ox-LDL stimulation.

A new question that arises from our study is how the PKC pathway regulates c-Fos expression. Previous studies have shown that PMA, an activator of PKC, stimulated c-fos promoter activity, transcriptional activity, and protein expression levels in intervertebral disc cells ${ }^{[43]}$. Whether mTORC2mediated PKC pathways regulate c-Fos at the transcriptional, translational, or post-translational levels in endothelial cells remains to be described.

It would be ideal if we could measure the expression of ICAM-1 and E-selectin using different approaches. Moreover, our research should be conducted in other cells, intact tissue or animals. In a recent study, we reported that rapamycin inhibits ox-LDL uptake in HUVECs. In this study, we showed that rapamycin inhibits ox-LDL-stimulated adhesion molecule expression and macrophage adhesion to HUVECs. Whether rapamycin has an effect on other processes of atherosclerosis warrants further study in the future.

In summary, we have demonstrated that rapamycin inhibits ox-LDL-stimulated adhesion molecule expression and macrophage adhesion to HUVECs by inhibiting mTORC2, but not mTORC1, and that mTORC2 acts through the PKC/c-Fos signaling pathway. Furthermore, mTORC2 and PKC may be potential therapeutic targets in atherosclerosis patients.

\section{Acknowledgments}

This study was supported by a grant from the Natural Sci- ence Foundation of Jiangsu Province (BK20122172 to Yan-ling. ZHANG), the National Natural Science Foundation of China (81200894 to Yan-ling ZHANG, 81471195 to Yong-jun CAO), a project funded by the Priority Academic Program Development of Jiangsu Higher Education Institutions and Suzhou Medical Key Discipline Project (to Chun-feng LIU), and support from the Preponderant Clinic Discipline Group Project Fund of the Second Affiliated Hospital of Soochow University (XKQ2015002).

\section{Author contribution}

Yong-jun CAO and Yan-lin ZHANG conceived and designed the study. Juan-juan SUN, Xiao-wei YIN, Hui-hui LIU and Wen-xiu DU performed the experiments. Juan-juan SUN, Xiao-wei YIN, Lu-yao SHI and Ya-bo HUANG analyzed the data. Juan-juan SUN, Xiao-wei YIN and Fen WANG wrote the paper. Chun-feng LIU, Yong-jun CAO and Yan-lin ZHANG reviewed and edited the manuscript. All authors read and approved the manuscript.

\section{References}

1 Libby P. Inflammation in atherosclerosis. Nature 2002; 420: 868-74.

2 Frostegård J, Wu R, Haegerstrand A, Patarroyo M, Lefvert AK, Nilsson J. Mononuclear leukocytes exposed to oxidized low density lipoprotein secrete a factor that stimulates endothelial cells to express adhesion molecules. Atherosclerosis 1993; 103: 213-9.

3 Ito F, Tatsumi H, Mori T, Suganuma I, Tanaka Y, Sasaki A, et al. Medroxyprogesterone acetate enhances monocyte-endothelial interaction under flow conditions by stimulating the expression of cell adhesion molecules. J Clin Endocrinol Metab 2014; 99: 2188-97.

4 Takahashi Y, Zhu H, Yoshimoto T. Essential roles of lipoxygenases in LDL oxidation and development of atherosclerosis. Antioxid Redox Signal 2005; 7: 425-31.

5 Guo YL, Bai R, Chen CX, Liu DQ, Liu Y, Zhang CY, et al. Role of junctional adhesion molecule-like protein in mediating monocyte transendothelial migration. Arterioscler Thromb Vasc Biol 2009; 29: 75-83.

6 Lewis JC, Kottle BA. Endothelial damage and thrombocyte adhesion in pigeon atherosclerosis. Science 1977; 196: 1007-9.

7 Shi Y, Frankel A, Radvanyi LG, Penn LZ, Miller RG, Mills GB. Rapamycin enhances apoptosis and increases sensitivity to cisplatin in vitro. Cancer Res 1995; 55: 1982-8.

8 Hosoi H, Dilling MB, Liu LN, Danks MK, Shikata T, Sekulic A, et al. Studies on the mechanism of resistance to rapamycin in human cancer cells. Mol Pharmacol 1998; 54: 815-24.

9 Sousa JE, Costa MA, Abizaid A, Sousa AG, Feres F, Mattos LA, et al. Sirolimus-eluting stent for the treatment of in-stent restenosis: a quantitative coronary angiography and three-dimensional intravascular ultrasound study. Circulation 2003; 107: 24-7.

10 Morice MC, Serruyys PW, Sousa JE, Fajadet J, Ban HE, Perrin M, et al. A randomized comparison of a sirolimus-eluting stent with a standard stent for coronary revascularization. N Engl J Med 2002; 346: 177380.

11 Hausleiter J, Kastrati A, Mehilli J, Vogeser M, Zohlnhöfer D, Schühlen $\mathrm{H}$, et al. OSIRIS Investigators. Randomized, double-blind, placebocontrolled trial of oral sirolimus for restenosis prevention in patients with in-stent restenosis: the oral sirolimus to inhibit recurrent in-stent stenosis (OSIRIS) trial. Circulation 2004; 110: 790-5.

12 Sehgal SN, Molnar-Kimber K, Ocain TD, Weichman BM. Rapamycin: a 
novel immunosuppressive macrolide. Med Res Rev 1994; 14: 1-22.

13 Laplante M, Sabatini DM. mTOR signaling in growth control and disease. Cell 2012; 149: 274-93.

14 Loewith R, Jacinto E, Wullschleger S, Lorberg A, Crespo JL, Bonenfant D, et al. Two mTOR complexes, only one of which is rapamycin sensitive, have distinct roles in cell growth control. Mol Cell 2002; 10: 457-68.

15 Kim D, Sarbassov D, Ali S., King J, Latek R, Erdjument Bromage H, et al. mTOR interacts with raptor to form a nutrient-sensitive complex that signals to the cell growth machinery. Cell 2002; 110: 163-75.

16 Hara K, Maruki Y, Long X, Yoshino K, Oshiro N, Hidayat S, et al. Raptor, a binding partner of target of rapamycin (TOR), mediates TOR action. Cell 2002; 110: 177-89.

17 Vander Haar E, Lee SI, Bandhakavi S, Griffi TJ, Kim DH. Insulin signalling to mTOR mediated by the Akt/PKB substrate PRAS40. Nat Cell Biol 2007; 9: 316-23.

18 Sancak Y, Thoreen CC, Peterson TR, Lindquist RA, Kang SA, Spooner E, et al. PRAS40 is an insulin-regulated inhibitor of the mTORC1 protein kinase. Mol Cell 2007; 25: 903-15.

19 Kim DH, Sarbassov DD, Ali SM, Latek RR, Guntur KV, ErdjumentBromage $\mathrm{H}$, et al. GbetaL, a positive regulator of the rapamycinsensitive pathway required for the nutrient-sensitive interaction between raptor and mTOR. Mol Cell 2003; 11: 895-904.

20 Sarbassov DD, Ali SM, Kim DH, Guertin DA, Latek RR, ErdjumentBromage $\mathrm{H}$, et al. Rictor, a novel binding partner of mTOR, defies a rapamycin-insensitive and raptor-independent pathway that regulates the cytoskeleton. Curr Biol 2004; 14: 1296-302.

21 Jacinto E, Loewith R, Schmidt A, Lin S, Ruegg MA, Hall A, et al. Mammalian TOR complex 2 controls the actin cytoskeleton and is rapamycin insensitive. Nat Cell Biol 2004; 6: 1122-8.

22 Sarbassov DD, Ali SM, Sengupta S, Sheen JH, Hsu PP, Bagley AF, et al. Prolonged rapamycin treatment inhibits mTORC2 assembly and Akt/ PKB. Mol Cell 2006; 22: 159-68.

23 Frias M, Thoreen C, Jaffe J, Schroder W, Sculley T, Carr S, et al. mSin1 is necessary for Akt/PKB phosphorylation, and its isoforms defie three distinct mTORC2s. Curr Biol 2006; 16: 1865-70.

24 Jacinto E, Facchinetti V, Liu D, Soto N, Wei S, Jung S, et al. SIN1/ MIP1 maintains rictor-mTOR complex integrity and regulates Akt phosphorylation and substrate specifiity. Cell 2006; 127: 25-37.

25 Yang Q, Inoki K, Ikenoue T, Guan K. Identifiation of Sin1 as an essential TORC2 component required for complex formation and kinase activity. Genes Dev 2006; 20: 2820-32.

26 Pearce LR, Huang X, Boudeau J, Pawlowski R, Wullschleger S, Deak $M$, et al. Identifiation of protor as a novel rictor-binding component of mTOR complex-2. Biochem J 2007; 405: 513-22.

27 Woo SY, Kim DH, Jun CB, Kim YM, Haar EV, Lee SI, et al. PRR5, a novel component of mTOR complex 2, regulates plateletderived growth factor receptor beta expression and signaling. J Biol Chem 2007; 282: 25604-12.

28 Oh WJ, Jacinto E. mTOR complex 2 signaling and functions. Cell Cycle 2011; 10: 2305-16.

29 Sarbassov DD, Guertin DA, Ali SM, Sabatini DM. Phosphorylation and regulation of Akt/PKB by the rictor-mTOR complex. Science 2005;
307: 1098-101.

30 Garcia-Martinez JM, Alessi DR. mTOR complex 2 (mTORC2) controls hydrophobic motif phosphorylation and activation of serum- and glucocorticoid-induced protein kinase 1 (SGK1). Biochem J 2008; 416: 375-85.

31 Liu L, Chen L, Chung J, Huang S. Rapamycin inhibits F-actin reorganization and phosphorylation of focal adhesion proteins. Oncogene 2008; 27: 4998-5010.

32 Liu L, Luo Y, Chen L, Shen T, Xu B, Chen W, et al. Rapamycin inhibits cytoskeleton reorganization and cell motility by suppressing RhoA expression and activity. J Biol Chem 2010; 285: 38362-73.

33 Zhao L, Ding T, Cyrus T, Cheng Y, Tian H, Ma M, et al. Low-dose oral sirolimus reduces atherogenesis, vascular inflammation and modulates plaque composition in mice lacking the LDL receptor. $\mathrm{Br} J$ Pharmacol 2009; 156: 774-85.

34 Baetta R, Granata A, M, Ferri N, Arnaboldi L, Bellosta S, Pfister P, et al. Everolimus inhibits monocyte/macrophage migration in vitro and their accumulation in carotid lesions of cholesterol-fed rabbits. J Pharmacol Exp Ther 2009; 328: 419-25.

35 Chen WQ, Zhong L, Zhang L, Ji XP, Zhang M, Zhao YX, et al. Oral rapamycin attenuates inflammation and enhances stability of atherosclerotic plaques in rabbits independent of serum lipid levels. Br J Pharmacol 2009; 156: 941-51.

36 Sawhney RS, Cookson MM, Sharma B, Hauser J, Brattain MG. Autocrine transforming growth factor alpha regulates cell adhesion by multiple signaling via specifi phosphorylation sites of p70S6 kinase in colon cancer cells. J Biol Chem 2004; 279: 47379-90.

37 Chen L, Xu B, Liu L, Liu C, Luo Y, Chen X, et al. Both mTORC1 and mTORC2 are involved in the regulation of cell adhesion. Oncotarget 2015; 6: 7136-50.

38 Wood SC, Bushar G, Tesfamariam B. Inhibition of mammalian target of rapamycin modulates expression of adhesion molecules in endothelial cells. Toxicol Lett 2006; 165: 242-9.

39 Lehle K, Schreml S, Kunz-Schughart LA, Rupprecht L, Birnbaum $\mathrm{DE}$, Schmid $\mathrm{C}$, et al. mTOR inhibitors and calcineurin inhibitors do not affect adhesion molecule expression of human macro- and microvascular endothelial cells. J Vasc Res 2008; 45: 333-42.

40 Wang C, Qin L, Manes TD, Kirkiles-Smith NC, Tellides G, Pober JS. Rapamycin antagonizes TNF induction of VCAM-1 on endothelialcells by inhibiting mTORC2. J Exp Med 2014; 211: 395-404.

41 Li L, Xu S, Yan J, Li Y, Wang X, Du R, et al. Mechanism of PKC activity affecting the adhesion reaction of endothelial cells with monocytes. Int J Cardiol 2015; 182: 361-7.

42 Ikenoue T, Inoki K, Yang Q. Essential function of TORC2 in PKC and Akt turn motif phosphorylation, maturation and signaling. EMBO J 2008; 27: 1919-31.

43 Yokoyama K1, Hiyama A, Arai F, Nukaga T, Sakai D, Mochida J. C-Fos regulation by the MAPK and PKC pathways in intervertebral disc cells. PLoS One 2013; 8: 73210.

44 Oliveira-Ferrer L, Rößler K, Haustein V, Schröder C, Wicklein D, Maltseva D, et al. c-FOS suppresses ovarian cancer progression by changing adhesion. Br J Cancer 2014; 110: 753-63. 